\title{
Priming of chords: Spreading activation or overlapping frequency spectra?
}

\author{
JAMSHED J. BHARUCHA and KEIKO STOECKIG \\ Dartmouth College, Hanover, New Hampshire
}

\begin{abstract}
A chord generates expectancies for related chords to follow. Expectancies can be studied by measuring the time to discriminate between a target chord and a mistuned foil as a function of the target's relatedness to a preceding prime chord. This priming paradigm has been employed to demonstrate that related targets are processed more quickly and are perceived to be more consonant than are unrelated targets (Bharucha \& Stoeckig, 1986). The priming experiments in the present paper were designed to determine whether expectancies are generated at a cognitive level, by activation spreading through a network that represents harmonic relationships, or solely at a sensory level, by the activation of frequency-specific units. In Experiment 1, prime-target pairs shared no component tones, but related pairs had overlapping frequency spectra. In Experiment 2, all overlapping frequency components were eliminated. Priming was equally strong in both experiments. We conclude that frequency-specific repetition priming cannot account for expectancies in harmony, suggesting that activation spreads at a cognitive level of representation.
\end{abstract}

Fundamental to music is the interplay between expectancies, as they unfold over time, and the varying degrees to which they are fulfilled or violated (e.g., Jones, 1981, 1982; Lerdahl \& Jackendoff, 1983; Meyer, 1956; Schenker, 1935/1979; Schoenberg, 1954/1969). Expectancies are the temporal bonds between events in a sequence and underlie their context-dependent characteristics. A chord is thus perceived as more consonant when it is strongly expected than when it is not (Bharucha \& Stoeckig, 1986). These sequential influences on consonance, and the patterns of expectation that spawn them, must be mediated by a processing system that continuously integrates musical information over time. Does this system involve a cognitive component that has-presumably through exposure-internalized regularities in harmony, or is it solely a sensory system that integrates frequency components over time?

In order to address this question, we developed a method for measuring harmonic expectancies using a priming task (Bharucha \& Stoeckig, 1986, Experiments 2 and 3). Subjects were presented with two chords in succession, the first called the prime and the second the target. The prime and target were either closely related (henceforth called related) or distantly related (henceforth called unrelated), using music-theoretic criteria for relatedness, substantiated empirically by relatedness judgments (Bharucha \& Krumhansl, 1983; Krumhansl, Bharucha, \& Castellano, 1982). We hypothesized that the prime would generate expectancies for related chords to follow, resulting in greater consonance and faster processing for related (relative to unrelated) targets.

The authors thank Carol Fowler, Howard Hughes, Carol Krumhansl, Caroline Palmer, Mark Schmuckler, Ernst Terhardt, and George Wolford for helpful comments on this research. Correspondence concerning this article should be addressed to J. J. Bharucha, Department of Psychology, Dartmouth College, Hanover, NH 03755.
In order to measure both consonance and processing time, subjects were instructed to discriminate, as fast as possible, between target chords and mistuned foils (generated by lowering the fifth degree by an eighth tone). Subjects were first given a training session in which they attained a criterion level of accuracy in discriminating between targets and foils, in the absence of a prime.

Targets were more likely to be correctly identified (i.e., judged in tune) and were identified more quickly when they were related than when they were unrelated to the prime. This facilitation of related targets (henceforth called a priming effect) was taken to be a robust demonstration of the anticipatory processing that underlies expectancies.

An examination of the foils revealed that the facilitation was primarily a biasing effect of the prime. Foils were less likely to be correctly identified (i.e., judged out of tune) and were identified more slowly when related than when unrelated to the prime. Thus the prime increases the consonance of related chords, making it easier to recognize that a related target is in tune and making it more difficult to recognize that a related foil is out of tune.

The prime also increases sensitivity to intonation for related chords, since intonation (in tune/out of tune) was correctly identified more often and more quickly for related than for unrelated chords.

We have proposed a connectionist model of harmonic expectancy (Bharucha, in press; Bharucha \& Stoeckig, 1986) that accounts for the priming results and a range of available data on the perception of harmony, including relatedness judgments and memory confusions (see Bharucha \& Krumhansl, 1983; Krumhansl, Bharucha, \& Castellano, 1982). In the model, cognitive units representing chords are linked to each other via units representing their parent keys. Hearing a chord causes its unit to be 
activated, possibly through frequency-specific channels (Deutsch, 1969). Activation spreads to its parent key units, then to their daughter chord units (reverberating back to the original chord units), and so on through the network, decaying with time. The spread of activation from one unit to another is determined by the strength of the link between them, strong links transmitting more activation than weak links. Link strengths reflect the perceived strength or stability of chords in their parent keys.

A chord sounded either harmonically or melodically generates a pattern of activation that radiates out from the sounded chord unit. In a priming experiment, related chord units receive more activation from the prime than do unrelated chord units, biasing the perception of related chords in the direction of greater consonance and facilitating the identification of related targets. It should be noted that the facilitation reflects differences in decision times resulting from different levels of target unit activation, not differences in the time for activation to spread (see Anderson, 1983; Ratcliff \& McKoon, 1981).

This model is consistent with expectation-based theories of music, most notably that of Meyer (1956). The pattern of activation in the network underlies the expectations for chords to follow. The relationship between the activation of a chord unit and the pattern of activation of the network as a whole provides a mechanism for accounting for the distinctive perceptual quality of a chord as a function of its prior context.

Activation spreading through a network is more difficult to establish for music than it is for language. Related prime-target pairs in music may be acoustically related; relatively low-level processes may thus be sufficient to recognize relatedness. In contrast, related prime-target pairs in semantic priming are not acoustically or orthographically related, but only related in meaning. The present series of experiments were motivated by the possibility of an alternative explanation of musical priming that is more ecological and parsimonious than spreading activation.

A strong candidate for an alternative explanation is frequency-specific repetition priming. The related primetarget pairs used by Bharucha and Stoeckig (1986) shared a component tone, whereas the unrelated pairs did not. For example, if the prime was $\mathrm{C}$ major (whose component tones are $C, E$, and $G$ ), the related target was G major (whose component tones are G, B, and D) and the unrelated target was $\mathrm{F}$ major (whose component tones are $F(A$, and $C H$ ). Repetition priming of the tone shared by related prime and target could have caused the observed priming of chords, eliminating the need to posit a network linking related chords.

We report a pair of experiments designed to decide between these two hypotheses about the processes responsible for chordal expectancies. The first hypothesis, which we call the spreading activation hypothesis, is that expectancies are generated by a cognitive system that has internalized the sequential regularities in music through prior exposure. The second hypothesis, which we call the overlapping spectra hypothesis, is that expectancies are generated at a fairly peripheral level by frequency-specific sensory units that are activated by the spectral components in the acoustic stimulus.

In Experiment 1, we employed chord pairs with no component tones in common. In Experiment 2, we removed all overlapping components of the harmonic spectra of the two chords. An absence of priming under these conditions would be evidence against the spreading activation hypothesis and would eliminate the need to posit cognitive structures that encode regularities. However, any residual priming effect would be evidence in favor of such structures.

On the basis of the results of our earlier experiments (Bharucha \& Stoeckig, 1986), we predicted that targets would be correctly identified more quickly when related than when unrelated to the prime. Since this difference is largely a criterion effect, we predicted the opposite for the foils, resulting in an interaction between relatedness and intonation.

\section{EXPERIMENT 1}

Since maximally related chords (those closest along the network) necessarily share component tones, the related pairs we employed were further apart along the network. Unrelated pairs were maximally distant along the network. For example, if the prime was $\mathrm{C}$ major (whose component tones are $C, E$, and $G$ ), the related chord was $\mathrm{Bb}$ major (whose component tones are $\mathrm{Bb}, \mathrm{D}$, and $\mathrm{F}$ ) and the unrelated chord was $F$ major (whose component tones are $F \$, A \#$, and $C \$$ ).

\section{Method}

Subjects. Thirteen introductory psychology students received partial course credit for participating as subjects. Number of years of musical training, as measured by years of instrumental instruction, ranged from 0 to 9 , with a mean of 4.3 and a median of 4 . All subjects reported having normal hearing, and only 1 reported having absolute pitch.

Apparatus. Chords were synthesized by an Apple Macintosh microcomputer and presented to subjects through a Sansui A-707 amplifier and Sennheiser HD-410 headphones.

Stimuli. Primes and targets were major chords based on an equaltempered tuning (in which one semitone is a frequency factor of $2^{1 / 12}$ ) based on an $A$ of $440 \mathrm{~Hz}$. Foils were mistuned versions of targets produced by lowering the frequency of the fifth degree by a factor of $2^{1 / 4 s}$ (an eighth tone). All chords were constructed by imposing an envelope over a five-octave range (starting at $65.41 \mathrm{~Hz}$ ), tapering off to the loudness threshold at each end. The 3 component tones of each chord were sampled from each of these five octaves and added in proportion to their envelope amplitudes (see Krumhansl, Bharucha, \& Kessler, 1982; Shepard, 1964). The waveform of each of the 15 component tones contained the first four harmonics with equal amplitudes.

Each of the 12 major chords occurred twice as a prime, followed by each of the following: related target, unrelated target, related foil, and unrelated foil. Each subject received the 96 trials in a different random order.

The chord pairs, specified in terms of distance between their roots, were as follows. Relative to the prime, the related chord was 10 
semitones higher (or, equivalently, 2 semitones lower), and the unrelated chord was 6 semitones higher (or, equivalently, 6 semitones lower). For example, when the prime was $\mathrm{C}$ major, the related chord was Bbmajor and the unrelated chord was $\mathrm{F}$ major.

Procedure. All subjects took part in a training session consisting of three examples each of in-tune and out-of-tune chords, followed by 48 trials in which they were instructed to distinguish between in-tune and out-of-tune chords. Feedback was given after each trial. The subjects were required to achieve an accuracy of at least $85 \%$ to proceed to the main experiment and were permitted to repeat the training session until the criterion was reached.

In the main experiment, the subjects initiated each trial by pressing the space bar. A trial began with a sequence of 16 tones of random pitch, lasting $2 \mathrm{sec}$ in all, designed to mask the influence of the previous trial. After a $1-\mathrm{sec}$ pause, the prime sounded for $3 \mathrm{sec}$, im mediately followed by a chord that sounded for $2 \mathrm{sec}$. The subjects were instructed to decide, "as quickly as possible, while being as accurate as possible," whether the second chord was in tune (a target) or out of tune (a foil) and to respond by pressing the appropriately marked key. The subjects were told after each response whether it was correct or incorrect. In addition, they received summary feedback about accuracy and mean reaction time after every 24 trials.

The subjects were given 12 practice trials (randomly drawn from experimental trials) at the beginning of the session, with additional practice if requested. Immediately following the experiment, the subjects were debriefed and asked to complete musical background questionnaires. The entire session lasted about $45 \mathrm{~min}$.

\section{Results}

Reaction times greater than 2,000 msec or less than 0 msec were discarded. Reaction times and error rates were each analyzed using an analysis of variance with two factors: relatedness (related vs. unrelated) and intonation (in tune vs. out of tune).

Mean reaction times (for correct responses) and error rates are shown in Figure 1. Relatedness and intonation interact as predicted $[F(1,12)=10.12, p=.008$, for reaction times; $F(1,12)=6.00, p=.029$, for errors]: subjects were faster and made fewer errors for related than for unrelated targets, and were slower and made more errors for related than for unrelated foils, indicating a bias to respond "in tune" when chords were related and "out of tune" when they were unrelated.

Overall reaction times were faster when chords were related than when they were unrelated, as indicated by a main effect of relatedness $[F(1,12)=15.42, p=.002]$, indicating an overall facilitation of related chords. However, overall error rates for related and unrelated chords did not differ.

The size of the priming effect (i.e., the difference in reaction time between related and unrelated targets) was not correlated with musical training $(r=.12)$.

\section{Discussion}

Subjects were faster and made fewer errors in identifying target chords when they were related than when they were unrelated to the prime. The reverse was true for the foils, indicating that the observed facilitation resulted primarily from a biasing effect of the prime: chords were more likely to be judged in tune when related than when unrelated. The prime also increased sensitivity to related
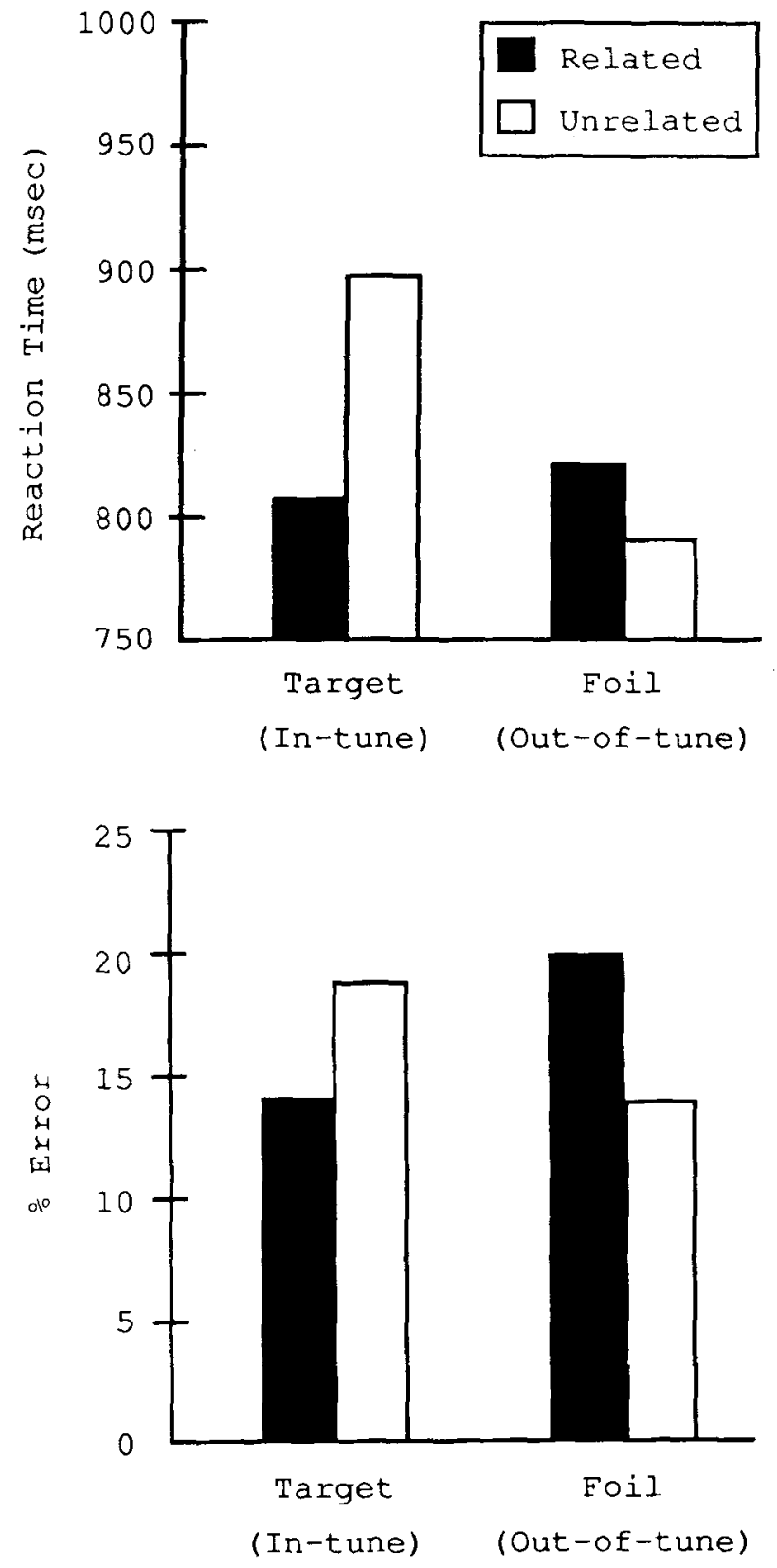

Figure 1. Priming of chords when related prime and target do not share component tones.

targets: subjects were faster and made fewer errors for related chords overall.

The related prime-target pairs did not share component tones, thus ruling out repetition priming of component tones as the sole explanation for the observed priming. However, the related prime-target pairs shared frequencies other than the fundamental frequencies of the component tones, since the component tones contained the first four harmonics. For example, the third harmonic of tone $G$ (a component tone of $C$ major) coincides with octave 
harmonics of $\mathrm{D}$, a component tone of the related $\mathrm{Bb}$ major chord. No such overlap existed between the harmonic spectra of unrelated prime-target pairs. The observed priming could thus have been caused solely by frequencyspecific repetition priming of upper harmonics.

\section{EXPERIMENT 2}

In the next experiment, we stripped the tones of their non-octave harmonics, thereby eliminating any overlap between the harmonic spectra of the two chords.

\section{Method}

Subjects. Fifteen introductory psychology students received partial course credit for participating as subjects. Number of years of musical training, as measured by years of instrumental instruction, ranged from 0 to 17 , with a mean of 5.2 and a median of 4 . All subjects reported having normal hearing, and only 1 reported having absolute pitch.

Apparatus. The apparatus was the same as in Experiment 1.

Stimuli. The stimuli were the same as in Experiment 1, except that the third harmonic was omitted from each component tone.

Procedure. The procedure was the same as in Experiment 1.

\section{Results}

Reaction times greater than 2,000 msec or less than $0 \mathrm{msec}$ were discarded. Reaction times and error rates were each analyzed using an analysis of variance with two factors: relatedness (related vs. unrelated) and intonation (in tune vs. out of tune).

Mean reaction times (for correct responses) and error rates are shown in Figure 2. Relatedness and intonation interact as predicted $[F(1,14)=8.81, p=.01$, for reaction times; $F(1,14)=6.95, p=.02$, for errors]: subjects were faster and made fewer errors for related than for unrelated targets, and were slower and made more errors for related than for unrelated foils, indicating a bias to respond "in tune" when related and "out of tune" when unrelated.

Overall, subjects were faster and made fewer errors when prime and target were related than when they were unrelated, but the differences were not significant. The pattern of results was thus the same as in Experiment 1, except that the sensitivity advantage for related targets was not significant.

The priming effect was not correlated with musical training $(r=-.05)$.

\section{Discussion}

Once again, subjects were faster and made fewer errors in identifying target chords when they were related than when they were unrelated to the prime. The opposite effect for the foils indicates that the observed facilitation resulted primarily from a biasing effect of the prime.

The impoverished harmonic spectra in Experiment 2 seemed to slow down reaction times relative to those of Experiment 1, although not significantly so. The error rates were comparable across experiments. If, indeed, reaction times for the impoverished stimuli were slower,
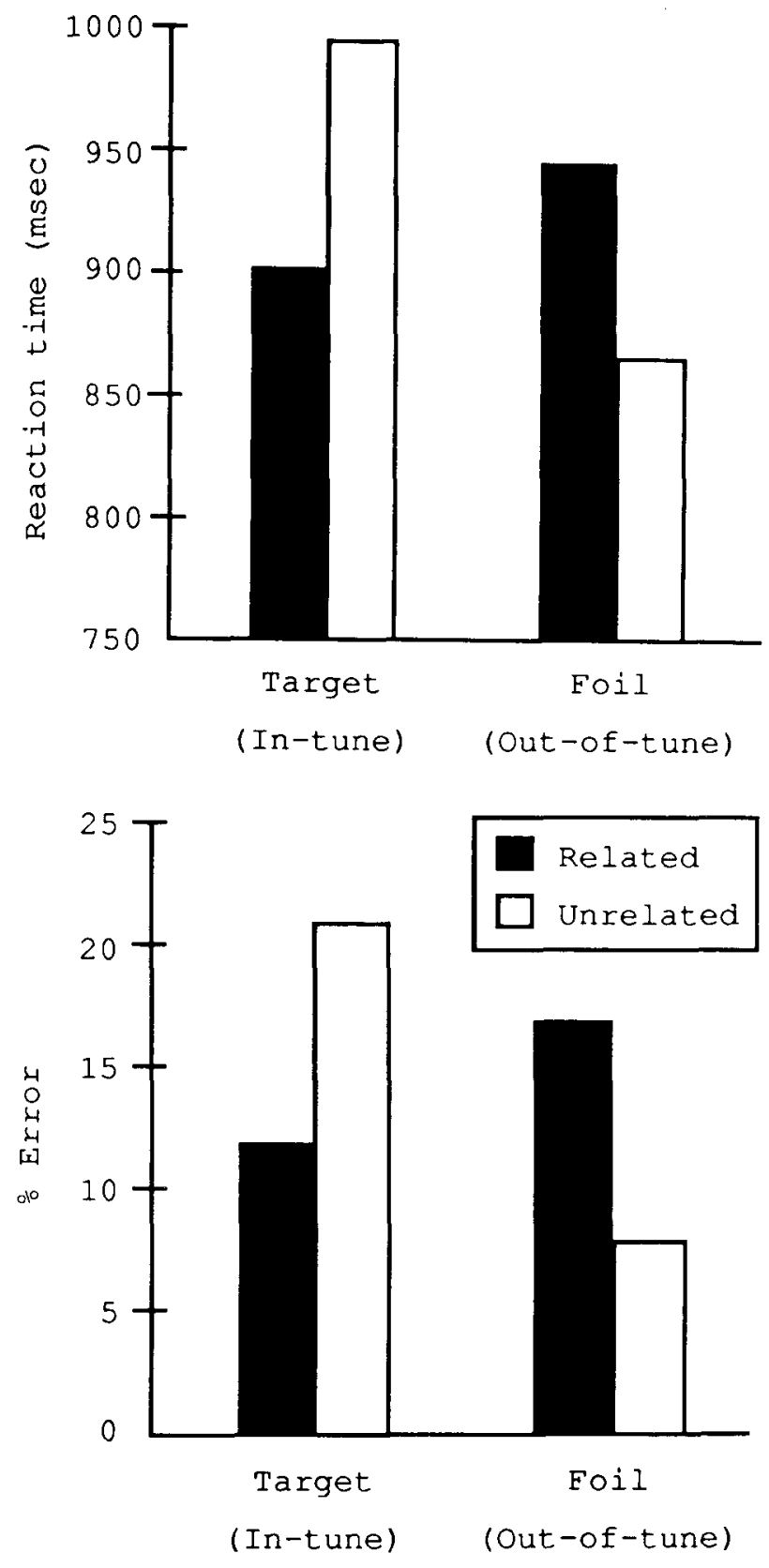

Figure 2. Priming of chords when the harmonic spectra of related prime and target do not overlap.

this would be consistent with the well established role of upper harmonics in sensory consonance (Helmholtz, 1863/1954; Levelt, van de Geer, \& Plomp, 1966; Terhardt, 1984). However, upper harmonics seemed to play a minimal role in priming. Although the slight sensitivity advantage for related over unrelated targets in Experiment 1 was not significant in Experiment 2, the size of the priming effect was roughly the same $(89 \mathrm{msec}$ for Experiment 1;96 msec for Experiment 2). There was no hint of either an experiment $\times$ relatedness interaction or an experiment $\times$ relatedness $\times$ intonation interaction. 
Thus there was little evidence that removing the overlapping spectral components reduced the priming effect.

\section{GENERAL DISCUSSION}

Related chords primed each other even when their frequency spectra did not overlap. A context can thus facilitate the perception of chords with entirely different frequency components. Barring nonlinear distortion in the ear, priming of specific frequencies cannot account for the observed priming of chords. This is evidence for activation spreading via a cognitive structure that links related chords.

We have outlined a parallel, interactive model (Bharucha, in press; Bharucha \& Stoeckig, 1986) in which the observed facilitation of related chords is the result of elements in a network activating each other via links between them. The pattern of connections and the strengths of the links constitute an internalization of chordal patterns that are pervasive in the Western musical environment. The pattern of activation after any given event in a sequence represents the array of expectancies. These expectancies, confirmed by reaction time measures, constitute anticipatory processing of events that are most likely to follow. Highly expected events, should they occur, are heard as more consonant than are highly unexpected events. The same processes can account for rating judgments and memory confusions as obtained in a number of studies (Bharucha \& Krumhansl, 1983; Cuddy, Cohen, \& Miller, 1979; Dowling, 1978; Krumhansl, 1979; Krumhansl, Bharucha, \& Castellano, 1982; Krumhansl \& Kessler, 1982). The higher the activation of a unit, the higher the rating judgment of the event represented by that unit, and the greater its confusability with the prior context (see Bharucha, in press).

In the absence of evidence that this internalized structure is innate, we assume it is the consequence of a general-purpose connectionist architecture that internalizes regularities in the environment. This, of course, raises the question why these chordal regularities occur in the first place. Basic psychoacoustic factors, such as sensory consonance resulting from overlapping spectra (Helmholtz, 1863/1954) and the fundamental bass resulting from virtual pitch phenomena (Terhardt, 1974, 1984), account remarkably well for the evolution of constraints on the simultaneous sounding of tones as chords. However, the evolution of sequential constraints on chords is more difficult to establish; in addition to basic psychoacoustic factors, pitch proximity relationships (Bharucha, 1984; Deutsch, 1978) and the resulting constraints on "voice leading'" may have been important. However, whatever the reasons for the pervasiveness of extant sequential constraints on chords, cognitive structures are formed that generate schematic expectancies, even when the factors that may have bootstrapped these constraints are absent.

The lack of correlation between musical training and the size of the priming effect points to the robustness of the processes being tapped. The internalization of musical structure does not come from explicit musical training, but presumably from mere exposure. The untrained subjects had little or no explicit knowledge of musical structure (as determined by musical background questionnaires administered to subjects), yet must have had considerable tacit knowledge. Perceptual learning of this sort can be accomplished by incremental changes in link strengths over time (Rumelhart \& McClelland, 1986). According to this view, music perception is a natural consequence of a general-purpose cognitive architecture shaped by regularities in the auditory environment.

\section{REFERENCES}

Anderson, J. R. (1983). The architecture of cognition. Cambridge, MA: Harvard University Press.

BharuCha, J. J. (1984). Anchoring effects in music: The resolution of dissonance. Cognitive Psychology, 16, 485-518.

Bharucha, J. J. (in press). MUSACT: A connectionist model of musical harmony. Proceedings of the Ninth Annual Conference of the Cognitive Science Society. Hillsdale: NJ: Erlbaum.

BharUCha, J., \& KRUmhansL, C. L. (1983). The representation of har monic structure in music: Hierarchies of stability as a function of context. Cognition, 13, 63-102.

Bharucha, J. J., \& Stoeckig, K. (1986). Reaction time and musical expectancy: Priming of chords. Joumal of Experimental Psychology. Human Perception \& Performance, 12, 403-410.

Cuddr, L. L., Cohen, A. J., \& Miller, J. (1979). Melody recognition: The experimental application of musical rules. Canadian Journal of Psychology, 28, 148-157.

Deutsch, D. (1969). Music recognition. Psychological Review, 76, 300-307.

DeutsCH, D. (1978). Delayed pitch comparisons and the principle of proximity. Perception \& Psychophysics, 23, 227-230.

Dow LING, W. J. (1978). Scale and contour: Two components of a theory of memory for melodies. Psychological Review, 85, 341-354.

Helmholtz, H. (1954). On the sensations of tone (A. J. Ellis, Trans.). New York: Dover. (Original work published 1863)

JoNes, M. R. (1981). Music as a stimulus for psychological motion: Part I. Some determinants of expectancies. Psychomusicology, 1 , 34-51.

Jones, M. R. (1982). Music as a stimulus for psychological motion: Part II. An expectancy model. Psychomusicology, 2, 1-13.

KrumhansL, C. L. (1979). The representation of musical pitch in a tonal context. Cognitive Psychology, 11, 346-374.

Krumhansl, C. L., Bharucha, J., \& Castellano, M. A. (1982). Key distance effects on perceived harmonic structure in music. Perception \& Psychophysics, 32, 96-108.

Krumhansl, C. L., Bharucha, J. J., \& Kessler, E. J. (1982). Perceived harmonic structure of chords in three related musical keys. Journal of Experimental Psychology: Human Perception \& Performance, 8, 24-36

Krumhansl, C. L., \& Kessler, E. J. (1982). Tracing the dynamic changes in perceived tonal organization in a spatial representation of musical keys. Psychological Review, 89, 334-368.

LERDAHL, F., \& JACKENDOFF, R. (1983). A generative theory of tonal music. Cambridge, MA: MIT Press.

Levelt, W. J. M., van de Geer, J. P., \& Plomp, R. (1966). Triadic comparisons of musical intervals. British Journal of Mathematical \& Statistical Psychology, 19, 163-179.

MEYER, L. (1956). Emotion and meaning in music. Chicago: University of Chicago Press.

RatCliff, R., MCKoon, G. (1981). Does activation really spread? Psychological Review, 88, 454-462.

Rumelhart, D. E., \& McClelland, J. L. (1986). Parallel distributed 
processing: Explorations in the microstructure of cognition (Vol. 1). Cambridge, MA: MIT Press.

SChenKer, H. (1979). Free composition (E. Oster, Ed. and Trans.). New York: Longman. (Original work published 1935)

SCHOENBERG, A. (1969). Structural functions of harmony (L. Stein, Ed.). New York: Norton. (Original work published 1954)
ShePard, R. N. (1964). Circularity in judgments of relative pitch. Journal of the Acoustical Society of America, 36, 2346-2353.

Terhard, E. (1974). Pitch, consonance, and harmony. Journal of the Acoustical Society of America, 55, 1061-1069.

Terhardt, E. (1984). The concept of musical consonance: A link between music and psychoacoustics. Music Perception, 1, 276-295. 